\title{
The stellar content of the ring in NGC $660^{\star}$
}

\author{
G. M. Karataeva ${ }^{1,3}$, N. A. Tikhonov ${ }^{2,4}$, O. A. Galazutdinova ${ }^{2,4}$, V. A. Hagen-Thorn ${ }^{1,3}$, and V. A. Yakovleva ${ }^{1,3}$ \\ 1 Astronomical Institute, St. Petersburg State University, Universitetsky pr., 28 Petrodvoretz, St. Petersburg 198504, Russia \\ 2 Special Astrophysical Observatory, N. Arkhyz, Karachai-Circassian Rep. 369167, Russia \\ ${ }^{3}$ Isaac Newton Institute of Chile, St. Petersburg Branch \\ ${ }^{4}$ Isaac Newton Institute of Chile, SAO Branch
}

Received 15 January 2004 / Accepted 27 February 2004

\begin{abstract}
We present the results of stellar photometry of the polar-ring galaxy NGC 660 using the Hubble Space Telescope's archival data obtained with the Wide Field Planetary Camera 2. The final list of the resolved stars contains 550 objects, a considerable part of which are blue and red supergiants belonging to the polar ring. The analysis of the Colour-Magnitude Diagram for polar ring stars shows that it is best represented by the isochrones with metallicity $Z=0.008$. The process of star formation in the polar ring was continuous and the age of the youngest detected stars is about $7 \mathrm{Myr}$.
\end{abstract}

Key words. galaxies: individual: NGC 660 - galaxies: peculiar - galaxies: starburst - galaxies: stellar content

\section{Introduction}

NGC 660, known for a long time as a peculiar galaxy containing two inclined dust lanes (Benvenuti et al. 1976), was included by Whitmore et al. (1990) in their catalogue of PolarRing Galaxies (PRGs), candidates and related objects as a possible candidate (C-13). The existence of two different kinematic systems was established from radio HI (Gottesman \& Mahon 1990) and CO (Combes et al. 1992) observations, and the galaxy was classified as a kinematically confirmed PRG (Arnaboldi \& Galletta 1993).

An extensive study of NGC 660 was performed by van Driel et al. (1995). The authors give a detailed description of the host galaxy (which is spiral, while host galaxies in PRGs are usually lenticular or elliptical) and its ring, which is not polar but is inclined to the disc of the host galaxy by $55^{\circ}$. The authors notice that the ring is very massive and may be stable (Sparke 1986). Multicolour optical observations made with Schmidt telescope with low resolution (5'.5) show that the ring is blue $\left(V-I_{\mathrm{c}}=1{ }^{\mathrm{m}} 0\right)$. From comparison with models of single burst star formation, the authors claim that the age of the ring is about 2 Gyr. However, many HII regions (Young et al. 1988; Armus et al. 1990) observed in the ring and the high mass of molecular gas sufficient for active star formation (Combes et al. 1992) may point to more recent star formation. Detecting blue supergiants in the ring may confirm this suggestion.

Send offprint requests to: G. Karataeva,

e-mail: narka@astro.spbu.ru

* Based on observations made with the NASA/ESA Hubble Space Telescope, obtained from the Space Telescope Science Institute, which is operated by the Association of Universities for Research in Astronomy, Inc., under NASA contract NAS5-26555.
Table 1. Observation log.

\begin{tabular}{ccr}
\hline \hline Date & Band & Exposure \\
\hline 04.07 .1995 & $F 660 W$ & $2 \times 80 \mathrm{~s}$ \\
03.07 .2001 & $F 450 W$ & $2 \times 230 \mathrm{~s}$ \\
& $F 814 W$ & $2 \times 230 \mathrm{~s}$ \\
\hline
\end{tabular}

The galaxy is one of the nearest PRGs $\left(D \sim 13 \mathrm{Mpc}, H_{0}=\right.$ $75 \mathrm{~km} \mathrm{~s}^{-1} \mathrm{Mpc}^{-1}$ ) and resolving its ring into stars probably may be achieved using HST images. Our study of PRGs NGC 2685 and NGC 4650A whose distances are further than NGC 660 confirms this possibility (Karataeva et al. 2004, Paper I). The HST archive contains several images of NGC 660, unfortunately not very deep. These images form the observational basis for our work devoted to resolving the ring of the galaxy into stars.

\section{Observations and basic data processing}

We use archival HST/WFPC2 data of NGC 660 (see Table 1). These observations were carried out as part of Proposals $\mathrm{N} 9042$ and N 5446. The data set consists of images $F 450 \mathrm{~W}$, $F 814 W, F 660 W$-bands with total exposure times of $460 \mathrm{~s}$, $460 \mathrm{~s}$ and $160 \mathrm{~s}$ accordingly. Unfortunately, these images cover only a part of the ring (see Fig. 1). The total size of NGC 660 according to RC3 is $8.3 \times 3 ! 2$. At the distance of NGC 660 the scale is $\simeq 65 \mathrm{pc}$ in $1^{\prime \prime}$.

The raw frames were processed with the standard WFPC2 pipeline. The data were extracted from the Archive using the On-The-Fly Reprocessing (OTFR) STScI archive system, which reconstructs and calibrates the original data with 


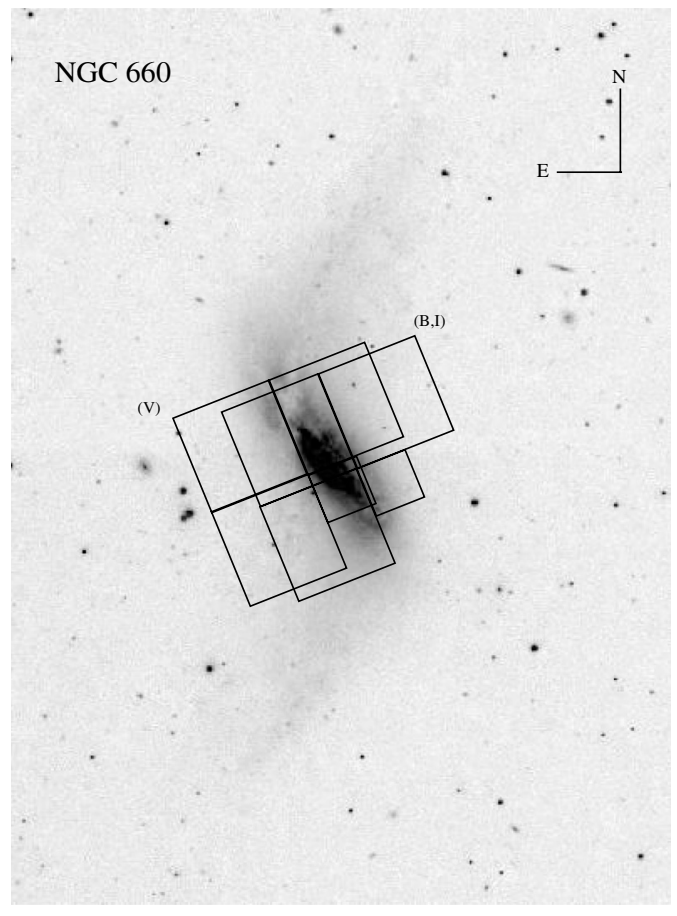

Fig. 1. DSS $28^{\prime} \times 11^{\prime}$ image of NGC 660 with WFPC2 footprint overlaid.

the latest calibration files, software and data parameters. For details of the processing see Paper I.

The single-star photometry of the images was processed with DAOPHOT/ALLSTAR. These programs use automatic starfinding algorithms and then measure the stellar magnitude by fitting a PSF that is constructed from the stars in uncrowded parts of the images. Then we determined the aperture correction from the 1.5 pixel radius aperture to the standard 0.5 radius aperture size for the WFPC2 photometric system using bright uncrowded stars. The $F 450 \mathrm{~W}, F 606 \mathrm{~W}$ and $F 814 W$ instrumental magnitudes were transformed to standard $B V I_{\mathrm{c}}$ in the Johnson-Cousins system using the prescriptions of Holtzman et al. (1995). Background galaxies, unresolved blends and stars contaminated by cosmetic CCD blemishes which have "quality" parameters as defined in ALLSTAR in MIDAS $|S H A R P|>0.1,|C H I|>1.0$ were eliminate from the final list which contains 550 stars.

The results of the artificial star completeness test are shown in Fig. 2, left. The recovery is practically complete up to $m=$ $23 \mathrm{~m} .5$ in $B$-band, $m=23.5$ in $V$-band and $m=24$. 0 in $I_{\mathrm{c}}$-band. Figure 2, right illustrates the precision of our photometry.

\section{Results}

The results of stellar photometry in the galaxy NGC 660 obtained after correcting for absorption in our Galaxy $\left(A_{B}=\right.$ 0. $28, A_{V}=0.21, A_{I_{\mathrm{c}}}=0 \mathrm{~m} 12$, Schlegel et al. 1998) are presented in Colour-Magnitude Diagrams (CMDs) in Fig. 3. For the CMD $B$ vs. $\left(B-I_{\mathrm{c}}\right)$ there is a concentration of blue stars in the region $0<\left(B-I_{\mathrm{c}}\right)<1$. 0 and 23 . $0<B<25^{\mathrm{m}} 0$. Taking into account that there must be intrinsic absorption in NGC 660 these stars may be considered as blue supergiants
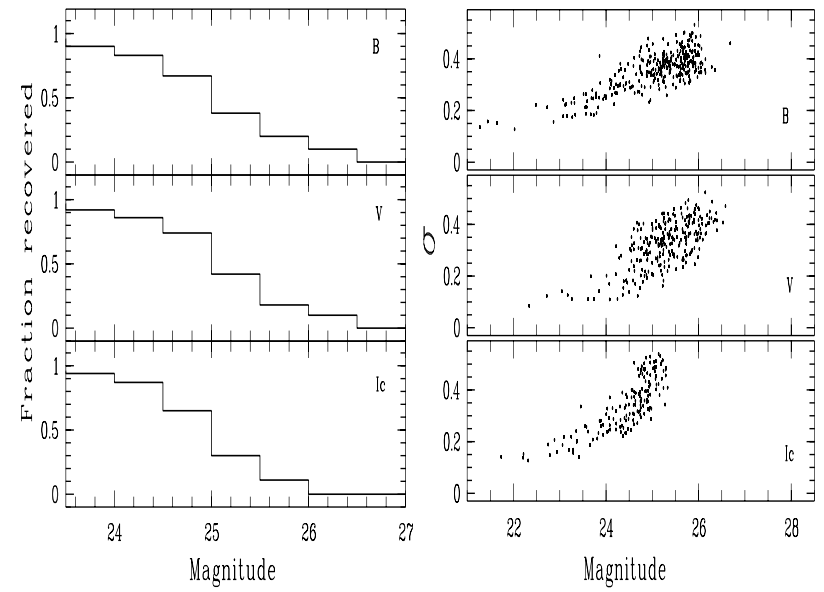

Fig. 2. Completeness levels (left) and errors (right) of the WFPC2 photometry.

by their colour indexes and luminosities (for NGC 660 the distance modulus is $m-M=30$. 6 ). The bulk of them are located in the region of the polar ring (Fig. 4).

While blue supergiants are detected in the CMD $B$ vs. $\left(B-I_{\mathrm{c}}\right)$ quite reliably (the normal position for blue supergiants can be found from Bertelli et al. 1994), the red supergiants, being near the limit in the $F 450 W$ image, are somewhat uncertainly. Nevertheless, some stars with $B<25.5$ and 2 . $8<\left(B-I_{\mathrm{c}}\right)<4 .{ }^{\mathrm{m}} 0$ are located in the ring. The $F 660 \mathrm{~W}$ image is more favorable for detecting red supergiants. Unfortunately, only a small part of the stars of the ring and host galaxy are represented in the CMD $I_{\mathrm{c}}$ vs. $\left(V-I_{\mathrm{c}}\right)$ (Fig. 3, right) because of the very short exposure time of the image as well as the large shift between the $F 660 \mathrm{~W}$ and $F 814 \mathrm{~W}$ images. However, one can see a concentration of stars in the region where red supergiants may be located $\left(I_{\mathrm{c}} \approx 22 \mathrm{~m} .5\right.$ and $\left.V-I_{\mathrm{c}} \approx 2 \mathrm{~m} 0\right)$. A part of these stars are located in the ring.

In Fig. 5, left we give the observed CMD $M_{I_{\mathrm{c}}}$ vs. $\left(B-I_{\mathrm{c}}\right)$ for all resolved stars constructed with the distance modulus $m-M=30^{\mathrm{m}} .6$. Absolute magnitudes of the blue stars are normal for blue supergiants, and taking into account their positions (Fig. 4) we have no reason not to consider them as ring stars. This is confirmed, in particular, by the location of some stars in complexes, two of which are presented in Fig. 4, right. One may expect that these complexes contain young stars. Such complexes were found in polar rings of the galaxies NGC 2685 and NGC 4650A (Paper I).

The large width of the blue supergiants branch $(0<(B-$ $\left.I_{\mathrm{c}}\right)<1$ ) in Figs. 3, left and 5, left may be explained by the different intrinsic absorption of various stars. The presence of dust in the ring may be expected from the large amount of neutral hydrogen observed therein (van Driel et al. 1995). The dust is seen directly as a dark strip in the region where the ring is projected on the disc of the galaxy. The dusty nature of the strip is confirmed by polarimetry (Reshetnikov \& Yakovleva 1991; Alton et al. 2000).

In the latter paper it is found that the absorption of the light of galactic disc by the ring is equal to 1.2 in the $V$-band (i.e. because $A_{\mathrm{V}}=-2.5 \lg \mathrm{e}^{-\tau_{\mathrm{v}}}$ the optical depth of dust layers in the ring is $\tau_{\mathrm{v}}=1.1$ ). This value gives the upper limit of 

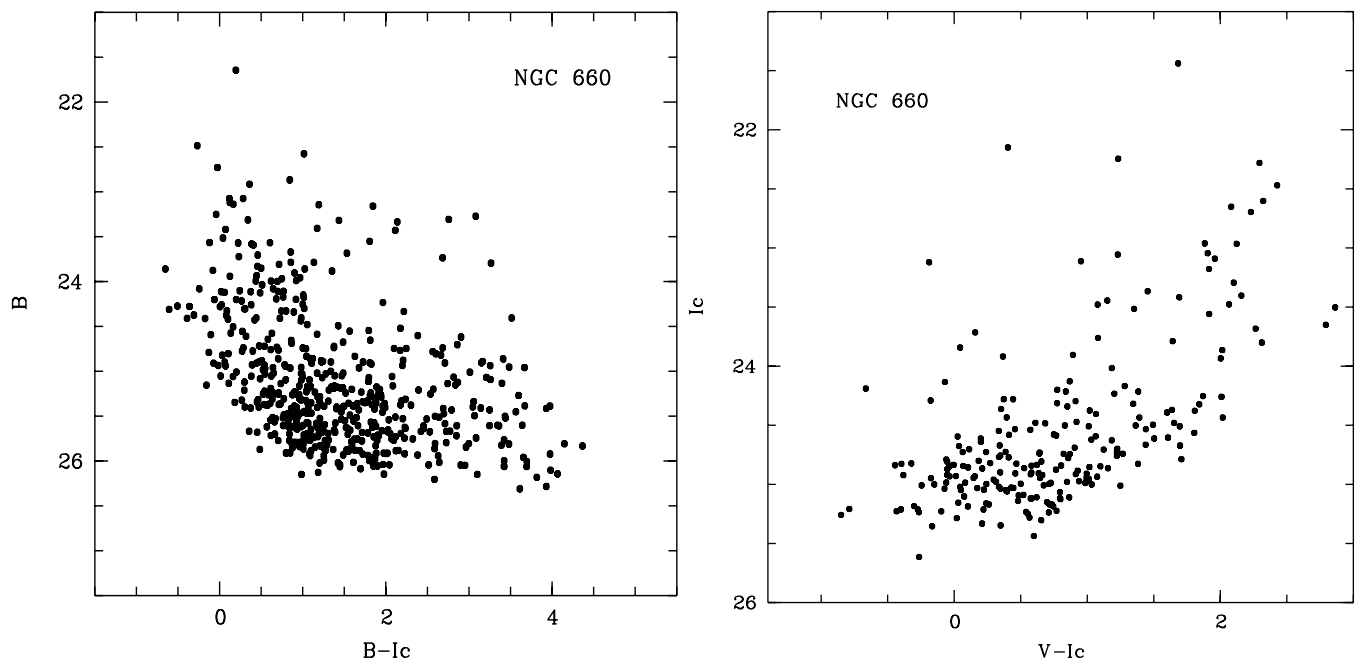

Fig. 3. Colour-Magnitude Diagrams $B$ vs. $\left(B-I_{\mathrm{c}}\right)($ left $)$ and $I_{\mathrm{c}}$ vs. $\left(V-I_{\mathrm{c}}\right)($ right $)$ corrected for absorption in our Galaxy.
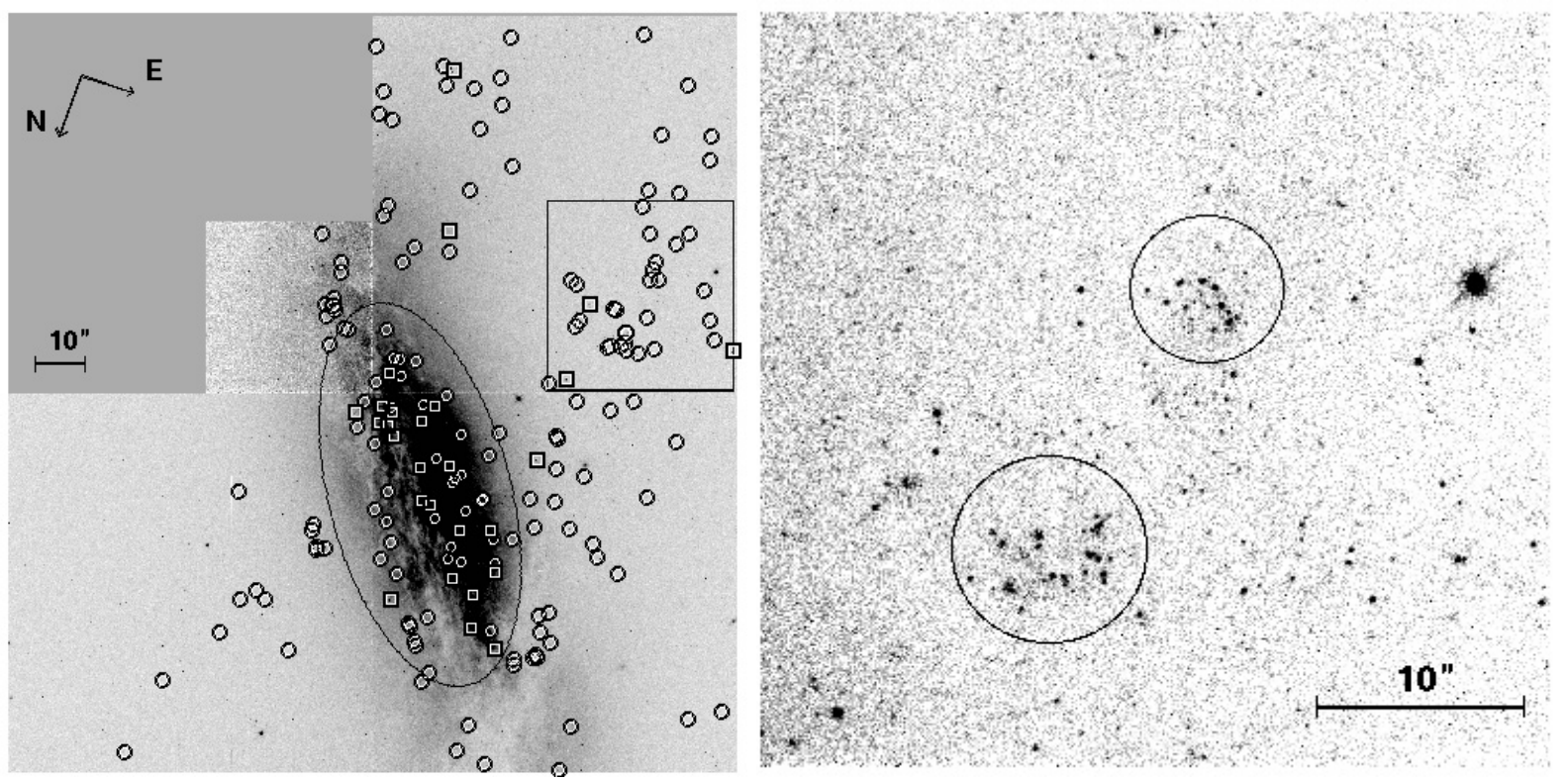

Fig. 4. WFPC2 image of NGC 660. Left: circles and squares indicate the position of blue and red supergiants, correspondingly. The stars located inside the ellipse were rejected in construction of Fig. 5, right. Right: two stellar complexes in the enlarged part (box on the left) of the NGC 660 polar ring.

absorption for the ring stars. Having no possibility to correct for absorption magnitudes and colour indexes of each star individually we correct CMDs for intrinsic absorption on average. If in the ring the dust and stars are mixed then for $\tau_{\mathrm{v}}=1.1$ one can find (Disney et al. 1989) the mean absorption for the ring stars of 0.54 . For a normal reddening curve (Cardelli et al. 1989) this gives $A_{B}=0.69, A_{I_{\mathrm{c}}}=0.34, E_{\left(B-I_{\mathrm{c}}\right)}=0.35$ and $E_{\left(V-I_{\mathrm{c}}\right)}=0 \mathrm{~m} 22$. These values may be used to correct CMDs.

Because our goal is to investigate the stellar content of the ring we should eliminate the stars not belonging to it from the final diagram. The final CMD for polar ring stars after correcting for intrinsic absorption and eliminating stars of the host galaxy (made geometrically, as shown in Fig. 4, left) is given in Fig. 5, right. Note that there is only a small difference between features in Figs. 5, right and left in the region of blue stars, which, therefore, belong to the ring. Of course, the CMD may contain some contaminating objects from our Galaxy (for instance, the object which has $M_{I_{\mathrm{c}}} \approx-10.8$ and $B-I_{\mathrm{c}} \approx 2 \mathrm{~m} 6$ ), but their number is not large.

\section{Discussion}

The CMD for ring stars (Fig. 5, right) may be used for estimation of their ages and metallicity by comparison with calculated isochrones. Bertelli et al. (1994) provide these isochrones for various metallicities. In Fig. 5, right isochrones for the metallicity $Z=0.008$ are overplotted on the CMD. Although the number of red supergiants is small, they allow us to choose between the isochrones with different metallicity. The isochrones for the metallicity $Z=0.008$ are in the best agreement with the observed CMD. The isochrones for higher metallicity (for example, solar, $Z=0.02$ ) contradict with the observed CMD 

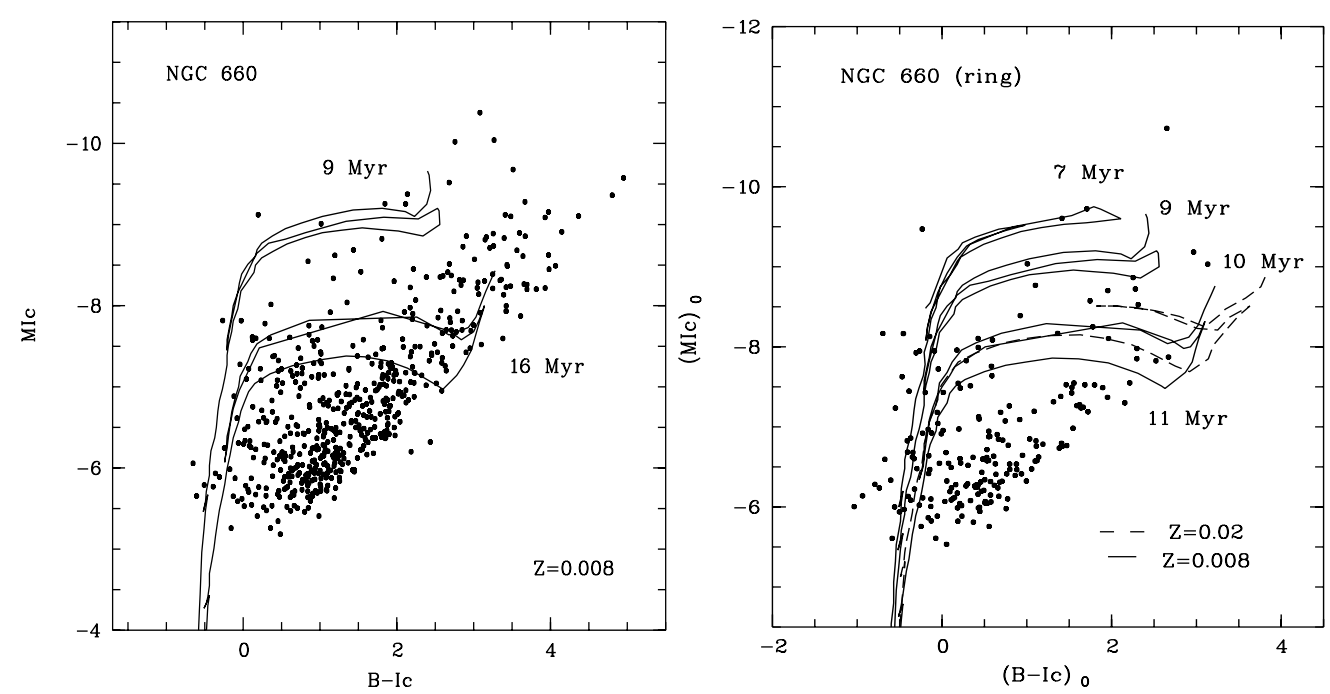

Fig. 5. $M_{I_{\mathrm{c}}}$ vs. $\left(B-I_{\mathrm{c}}\right)$ CMDs of NGC 660: left - for all resolved stars; right - for ring stars after correction for intrinsic absorption in the ring. Stellar isochrones for the metallicity $Z=0.008$ from the Padova library are overplotted. Dashed line on the right represents an example of a stellar isochrone for the metallicity $Z=0.02$.

(see Fig. 5, right). A relatively low metallicity in the polar rings was found for NGC 2685 (Paper I) and some other polar rings (Radtke et al. 2003). This is an important fact which should be taken into account in choosing a scenario of polar ring formation.

Figure 5, right shows that in the ring of NGC 660 there are very young stars with ages $7 \div 9$ Myr. Grouping the stars in complexes (see Fig. 4, right) shows that the stars are not yet dispersed in the stellar field, confirming their youth.

Because the CMD in Fig. 5, right is not deep, we cannot conclude anything about age of the ring. It may also contain old stars, like the Arp ring near M 81, where Tikhonov \& Galazutdinova (2004) detected red giants as well as young blue stars.

The relative youth of the ring is confirmed by its blue colour, as derived from surface photometry. Van Driel et al. (1995) showed that the ring with its colour index $V-I_{\mathrm{c}} \approx 1$. 0 is much bluer than the galaxy disc. Dividing frame $F 450 \mathrm{~W}$ by frame $F 814 W$ (see Fig. 6) confirms this. By comparison of this colour index with models of galactic colour evolution, van Driel et al. (1995) found that the age of the ring is about $2 \times 10^{9}$ years. However, the authors did not take into account any reddening. Alton et al. (2000) corroborated this and found that after correcting for intrinsic absorption (which they somewhat overestimated) the colour-index reduces to $V-I_{\mathrm{c}} \approx 0.7$ and age - to $\sim 10^{8}$ years (this value was found after an incorrect comparison of the colour index $V-I_{\mathrm{c}}$ (Cousins) with models, calculated for $V-I$ (Johnson)). Indeed, the full (Galactic and intrinsic) colour excess is $E_{V-I_{\mathrm{c}}}=0.31$ (see above) and correct comparison with the models calculated for $V-I_{\mathrm{c}}$ (Cousins) (Kurth et al. 1999) give the age of the ring as $1 \times 10^{9}$ years.

A final conclusion about the age of the ring may be obtained only after constructing the CMD sufficiently deep to reach the region of red giants. Now we can conclude only that very young stars exist in the ring and that the starforming process is continuous because in Fig. 5, right there are many stars below the isochrones.

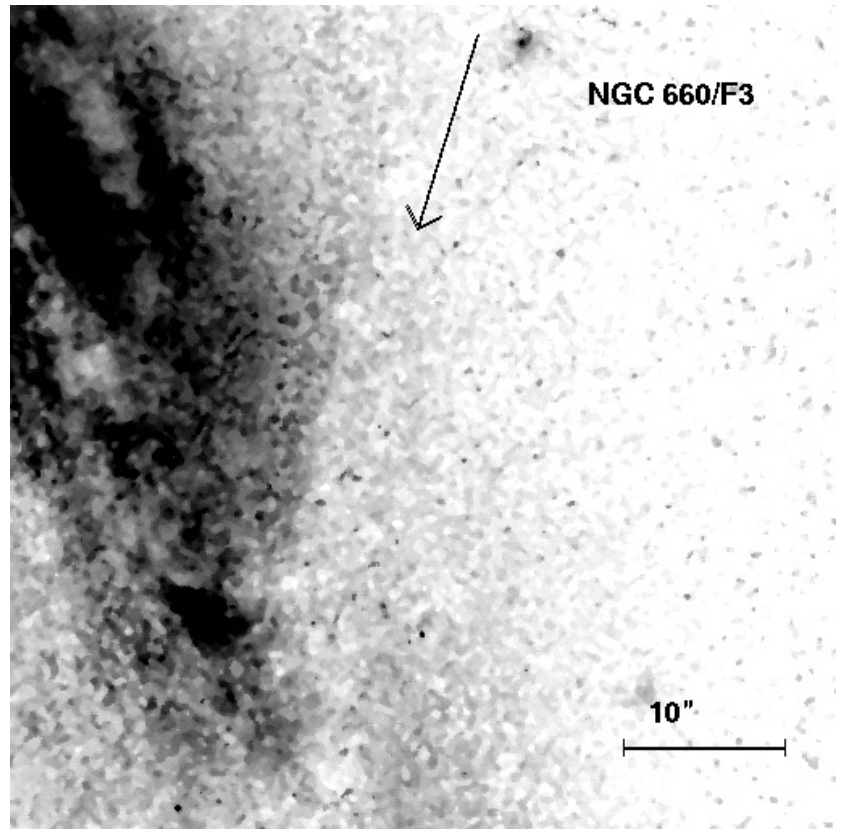

Fig. 6. The distribution of the colour $B-I_{\mathrm{c}}$ in NGC 660 (chip WF3) (the scale is such that dark to light is the sense red to blue). The arrow indicates the direction of polar ring.

\section{Conclusions}

We have resolved the ring of NGC 660 into stars. Some blue and red supergiants were found with ages of about $7 \div 9 \mathrm{Myr}$. The relatively low metallicity of these stars is in accordance with the data for polar rings in other PRGs.

It is very important to reach the region of red giants in CMDs. This might give not only the information about ring age but also assist in choosing the model of ring formation because the merging scenario (Bournaud \& Combes 2003) predicts the existence around the galaxy of a halo from old/intermediate age 
stars. Longer exposures with HST may allow us to construct such CMDs.

Acknowledgements. This work was supported by RFBR via grants 03-02-16344 and 02-02-16033.

\section{References}

Alton, P. B., Stockdale, D. P., Scarrott, S. M., \& Wolstencroft, R. D. 2000, A\&A, 357, 443

Armus, L., Heckman, T. M., \& Miley, G. K. 1990, ApJ, 364, 471

Arnaboldi, M., \& Galletta, G. 1993, A\&A, 268, 411

Benvenuti, P., Capaccioli, M., \& d'Odorico, S. 1976, A\&A, 53, 141

Bertelli, G., Bressan, A., Choisi, C., Fagotto, F., \& Nasi, E. 1994, A\&A, 106, 275

Bournaud, F., Combes, F. 2003, A\&A, 401, 817

Cardelli, J. A., Clayton, G. C., Mathys, J. S. 1989, ApJ, 345, 245

Combes, F., Braine, J., Casoli, F., Gerin, M., \& van Driel, W. 1992, A\&A, 259, L65

Disney, M., Davies, J., Phillips, S. 1989, MNRAS, 239, 939
Garnett, D. R. 2002, ApJ, 581, 1019

Gottesman, S. T., \& Mahon, M. E. 1990, in Paired and Interacting Galaxies, IAU Coll., 124, ed. J. W. Sulentic, W. C. Keel, \& C. M. Telesco, NASA Conf. Publ., 3098, 209

Eskridge, P. B., Pogge, R. W. 1997, ApJ, 486, 259

Holtzman, J. A., Burrows, C. J., Casertano, S., et al. 1995, PASP, 107, 1065

Karataeva, G. M., Drozdovsky, I. O., Hagen-Thorn, V. A., et al. 2004, AJ, 127, 789 (Paper I)

Kurth, O. A., Fritze-v. Alvensleben, U., Fricke, K. J. 1999, A\&A, 138, 19

Schlegel, D. J., Finkbeiner, D. P., \& Davis, M. 1998, ApJ, 500, 525 Sparke, L. 1986, MNRAS, 219, 657

Radtke, I. R., Eskridge, P. B., \& Pogge, R. W. 2003, A\&AS, 202, 4010

Reshetnikov, V. P., \& Yakovleva, V. A. 1991, Astrofizika, 35, 61

Tikhonov, N. A., \& Galazutdinova, O. A. 2004, in preparation

van Driel, W., Combes, F., Casoli, F., et al. 1995, AJ, 109, 942

Whitmore, B. C., Lucas, R. A., McElroy, D. B., et al. 1990, AJ, 100, 1489

Young, J. S., Kleinmann, S. G., \& Allen, L. E. 1988, ApJ, 334, L63 